\title{
Nonlinearity in color space measured by apparent motion
}

\author{
SATOSHI SHIOIRI \\ Chiba University, Chiba, Japan \\ and \\ PATRICK CAVANAGH \\ Harvard University, Cambridge, Massachusetts
}

\begin{abstract}
We used an apparent motion technique to examine the intensity coding along the three cardinal axes of color space: achromatic $(\mathrm{L}+\mathrm{M}+\mathrm{S}), \mathrm{L}-\mathrm{M}$ cone, and $\mathrm{S}$ cone axes. Two horizontal bars of different colors were alternated to produce a vertical displacement. The color of the background was a mixture varied between the colors of the two bars. When the background color was close to either of the test colors, only the bar that was more salient appeared to jump. Observers adjusted the color of the background until they saw either the two bars moved equally frequently or both bars moved at once. If the color difference in a linear cone excitation space controls this apparent motion, the setting should be midway between the two colors. All of the three cardinal axes showed some deviation from linear behavior. The nonlinearity was less extreme than a logarithmic function for both the achromatic and $\mathrm{S}$ cone axes and could be attributed to a small compressive nonlinearity, possibly at the level of cone responses. However, the $\mathrm{L}-\mathrm{M}$ stimuli showed a more extreme departure from linearity, which suggested a nonlinearity at an opponent site. A test of perceived contrast judgments did not show this nonlinearity for $\mathrm{L}-\mathrm{M}$ axis, suggesting that it is specific to the $\mathrm{L}-\mathrm{M}$ contribution to apparent motion.
\end{abstract}

What level of gray falls midway between black and white? In an early experiment, Plateau (1872) asked several painters to mix the mid-gray that they felt lay halfway between a black and a white paint. The gray chosen by the painters was much darker than the gray made by mixing equal parts of the black and white paints. In other words, it was not the arithmetic mean of the two intensities. If anything, it was more like the geometric mean, a value that makes the ratio between mid-gray and black equal to the ratio between white and mid-gray. Other studies in the 19th century came to similar conclusions (Delboeuf, 1883; Fechner, 1860/1966; Lehmann, 1886). One interpretation of this equal-ratio property is that there is an underlying logarithmic response to intensity, as pointed out by Fechner for his equal sensation scales and, much later, by Anstis and Mather (1985). However, another interpretation is that responses vary linearly with the difference but are scaled by the mean or adaptation level. This response relative to the mean will also lead to an equal-ratio property in the bisection task in which the

Support for this research was provided by MESSC Grant-in-Aid for Scientific Research (c) 10650036 to S.S. and by NEI Grant EY09258 to P.C. The authors thank Charles Stromeyer III for his helpful comments on the manuscript and Rand Evans for the 19th century references on brightness bisection. Correspondence should be addressed to S. Shioiri, Department of Information and Image Sciences, Chiba University, 1-33, Yayoi-cho, Inage-Ku, Chiba, 263-8522, Japan (e-mail: shiori@image.tp.chiba-u.ac.jp). three stimuli are compared two at a time leading to different mean levels in the two pairs. Other tasks, such as lightness scaling, have also shown a similar influence of the background level (Kaneko, 1964; Wyszecki \& Stiles, 1982).

Anstis and Mather (1985) introduced a new technique that made it possible to make the bisection judgment using all three values (light, dark, and mid-gray) at once. Their study revealed an underlying, almost linear response: The mid-gray fell at the arithmetic mean of black and white. In their procedure, two bars of different luminance (Figure 1) were displayed on a background of an intermediate value. When the two bars exchanged positions, only one of the bars appeared to move, even though, in fact, both bars had traded places. On a dim background, for example, only the lighter bar appeared to move; the black bar appeared as a fixed background region that was being covered and uncovered by the moving white bar. It was the bar that was most dissimilar to the background, which appeared to move (Figure 2). In Anstis and Mather's experiment, the luminance of the background was varied to determine the point at which neither bar appeared moving. Both bars evoke equal responses at that point. If the response is linear, the crossover point should occur when the background luminance is the arithmetic average of the luminance of the two bars. Anstis and Mather found that the equal response point was well predicted by linearity.

We extended their test to examine whether a linear contrast function was found along the two color axes and 


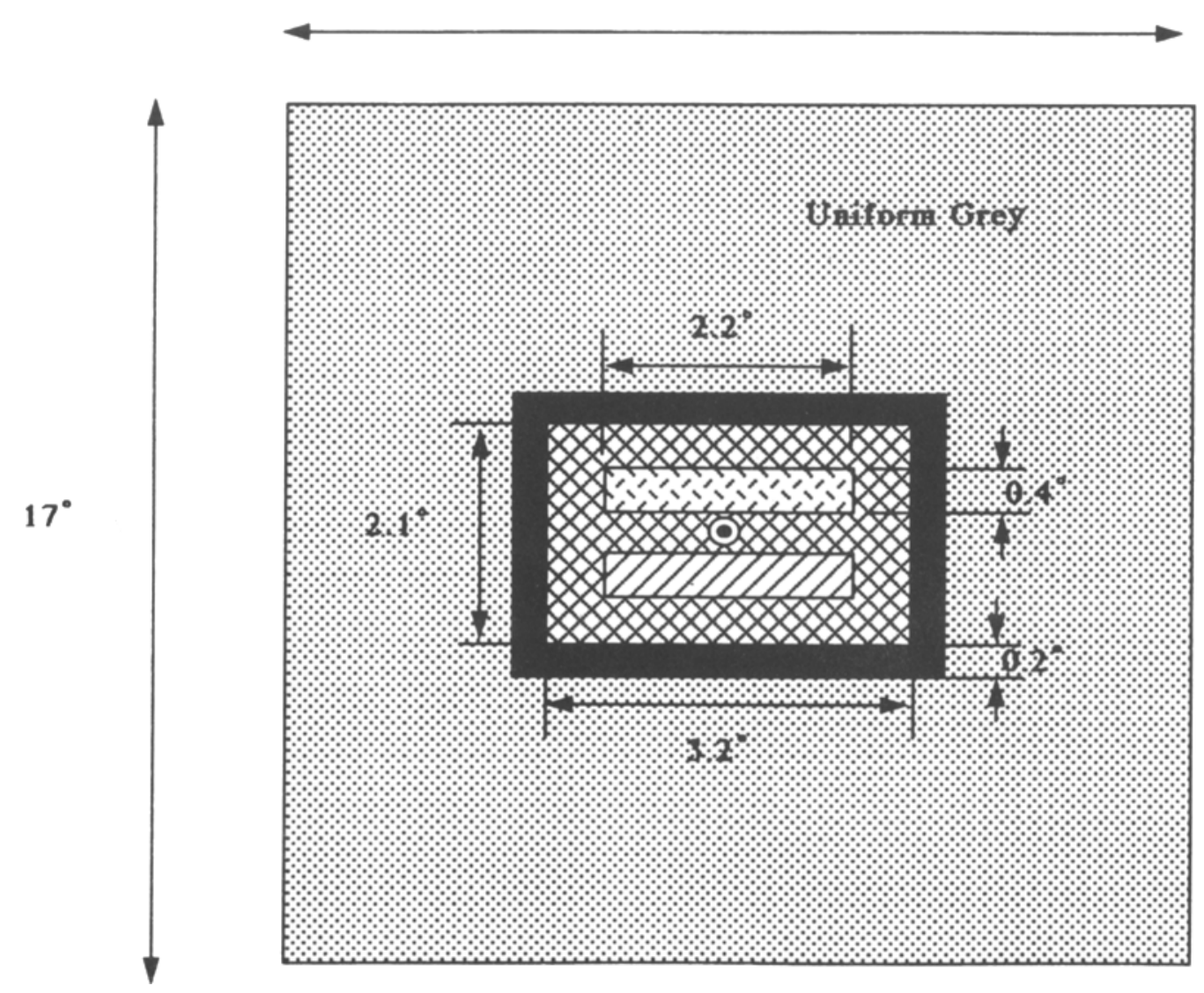

Figure 1. The stimulus configuration for the apparent-motion test. The colors of the two central horizontal bars exchanged location at $1 \mathrm{~Hz}$.

to reevaluate the linearity of Anstis and Mather's (1985) results along the achromatic axis. The color of the background was the mixture of the two colors with variable ratios. Typically, when the bars exchanged position, the bar with the color that was least like the background color was seen to move. The mixture ratio in the background was changed to determine the equal response point where either the two bars moved equally frequently or both bars moved at once. This point of equal motion should occur when the background is at the arithmetic midpoint between the two colors if the motion response to the different color values is linear.

We used cone excitation color space to represent our stimuli (Boynton, 1986; Derrington, Krauskopf, \& Len-

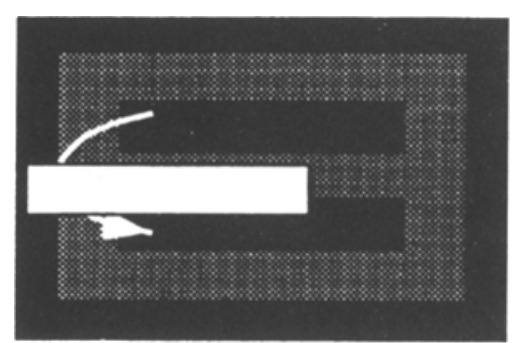

(a)

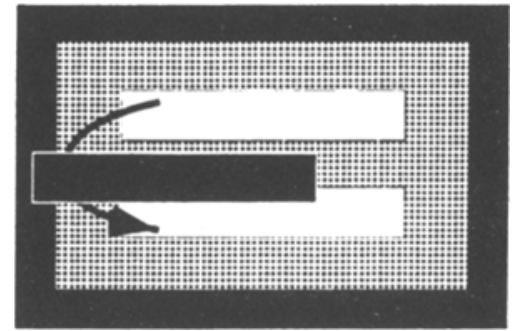

(b)

Figure 2. Although the two bars exchanged colors, for most settings, only one bar appeared to move as depicted here. When the background was dark, the white bar appeared to move (a), whereas when the background was lighter, the dark bar appeared to move (b). 
nie, 1984; Flanagan, Cavanagh, \& Favreau, 1990; MacLeod \& Boynton, 1979). The cone excitation color space used here is a three-dimensional linear space with coordinates of the sum of $L, M$, and $S$ cone excitation $(L+M+S$ or achromatic), the difference of $L$ and $M$ cone excitation (L-M) and S cone excitation (Flanagan et al., 1990). The linearity of the color scaling in this space was the question of the present study. Nonlinear scaling was expected because the cone response is known to have a compressive nonlinearity (Boynton \& Whitten 1970; Naka \& Rushton, 1966; Valeton \& van Norren, 1983), and equal perceived color steps in Munsell color space are not linearly related to the cone responses.

Finally, we ran a second experiment requiring observers to set the background of the two bars so that both bars had equal perceived contrast relative to the background. The purpose of this contrast judgment was to determine whether any nonlinearities we might find in the motion setting were specific to the apparent motion response.

\section{EXPERIMENT 1}

\section{Principle}

The linearity of the motion response to color can be tested by determining whether the mean of two colors lies halfway between them. Two bars of different colors $\left(\mathrm{C}_{\mathrm{a}}, \mathrm{C}_{\mathrm{b}}\right)$ are presented on a background of a mixture color $\left(\mathrm{C}_{\mathrm{bk}}\right)$ formed by the equation $\mathrm{C}_{\mathrm{bk}}=(k-1) \cdot \mathrm{C}_{\mathrm{a}}+k \cdot \mathrm{C}_{\mathrm{b}}$, where $k$ varies between 0 and 1 . If the response is linear, the difference in output (or response) between each of the bars and the background will be equal when the background color is midway between $\mathrm{C}_{\mathrm{a}}$ and $\mathrm{C}_{\mathrm{b}}(k=.5)$. For a nonlinear response, on the other hand, equal difference occurs for unequal mixtures of $\mathrm{C}_{\mathrm{a}}$ and $\mathrm{C}_{\mathrm{b}}$. For example, if the system has a compressive nonlinearity and, say, $\mathrm{C}_{b}$ is greater than $\mathrm{C}_{\mathrm{a}}$ along the axis, then $\mathrm{C}_{\mathrm{bk}}$ has to be closer to $\mathrm{C}_{\mathrm{a}}(k<.5)$ to obtain equal output differences.

This method can be used to determine the input-output relation along any axis in the color space ( $\mathrm{L}-\mathrm{M}, \mathrm{S}$ cone, or achromatic) using an incremental procedure like that used in bisection methods (Wyszecki \& Stiles, 1982). First, $\mathrm{C}_{\mathrm{a}}$ and $\mathrm{C}_{\mathrm{b}}$ are assigned normalized values of $0 \%$ and $100 \%$, respectively. Then, the value of $k$ for providing equal contrast against $\mathrm{C}_{a}$ and $\mathrm{C}_{\mathrm{b}}$ is labeled $\mathrm{C}_{50 \%}$ since it gives the midpoint response between $\mathrm{C}_{\mathrm{a}}$ and $\mathrm{C}_{\mathrm{b}}$. Then, using $\mathrm{C}_{50 \%}$ as one of the bar colors and either $\mathrm{C}_{\mathrm{a}}$ or $\mathrm{C}_{\mathrm{b}}$ as the other, the background color for equal amplitude against $\mathrm{C}_{\mathrm{a}}$ or $\mathrm{C}_{\mathrm{b}}$ can be determined. They are labeled $\mathrm{C}_{25 \%}$ and $\mathrm{C}_{75 \%}$. These colors are then used as one of the bar colors in the next step. Obtaining $\mathrm{C}_{12.5 \%}, \mathrm{C}_{37.5 \%}, \mathrm{C}_{62.5 \%}$, and so on, by this method gives input-output function over the range between $C_{a}$ and $C_{b}$.

\section{Method}

Stimuli were presented on a 19-in. Conrac 5411 RGB monitor, controlled by a Grinnell 270 color graphics system with $512 \times 480$ pixels spatial resolution, 256 intensity levels per color, and a $30-\mathrm{Hz}$ interlaced raster. Internal look-up tables in the Grinnell linearized the luminance output of each phosphor. The red, green, and blue phosphors of the monitor were determined by spectroradiometry to have CIE $x$ and $y$ coordinates of .611 and $.344, .301$ and .607 , and .151 and .074 , respectively. The screen was viewed from a distance of $90 \mathrm{~cm}$ and subtended a visual angle of $17^{\circ} \times 17^{\circ}$. The stimulus covered only a small area of the screen, $3.2^{\circ} \times 2.1^{\circ}$, to minimize the possible effect of screen inhomogeneity. Each horizontal bar was $2.2^{\circ}$ long and $0.4^{\circ}$ high, and the two bars were located at $0.4^{\circ}$ above and below the center (Figure 1). The outside of the stimulus field $\left(17^{\circ} \times 17^{\circ}\right)$ was filled with a uniform gray of $60 \mathrm{~cd} / \mathrm{m}^{2}$, separated from the stimulus field by a $0.2^{\circ}$ black gap.

The color pair was black of $7.0 \mathrm{~cd} / \mathrm{m}^{2}$ and white of $120 \mathrm{~cd} / \mathrm{m}^{2}$ for the achromatic axis. CIE $x$ and $y$ coordinates of the color pair for the $\mathrm{L}-\mathrm{M}$ cone axis were .247 and .370 (desaturated cyan) and .401 and .294 (desaturated magenta), and those for the S cone axis were .298 and .255 (purple) and .396 and .458 (greenish yellow). The luminance level for equiluminant color axes was $60 \mathrm{~cd} / \mathrm{m}^{2}$. After the linearization, the minimum step of the color change along each axis within the regions we were testing was less than $1 \%$ of the distance between the two colors at the ends of each axis. The L-M direction in the color plane is the direction specified by CIE coordinates and the Smith-Pokorny functions (Boynton, 1986). To set the equiluminant plane, each observer adjusted the luminance for red and blue phosphors by heterochromatic flicker photometry so that the desaturated magenta and purple had the same effective luminance for the observer as the desaturated cyan, which was maintained at $60 \mathrm{~cd} / \mathrm{m}^{2}$. Minimum flicker was set using the same two bars of the main experiment with the colors alternated at $15 \mathrm{~Hz}$. The background was fixed at white of $60 \mathrm{~cd} / \mathrm{m}^{2}$. The observers performed the setting by adjusting the individual contrast setting for the red and blue phosphors prior to each experimental session.

The bars exchanged positions periodically at $1 \mathrm{~Hz}(500 \mathrm{msec}$ for each position) while the observers adjusted the color of the background using a joystick. The adjustment was made to determine the point at which either the two bars move equally frequently or both bars move at once. The initial background color was arbitrarily set by the observers to produce a clear impression of apparent motion of only one bar. The observers viewed monocularly with the right eye, with natural pupil. No correction for chromatic aberration was made. Two observers with corrected-to-normal acuity participated in the experiment.

\section{Results}

Achromatic axis. Figure 3 shows the results for the achromatic axis (i.e., the black and white color pair). The horizontal axis indicates background luminance, and the vertical axis shows the normalized response (as is the convention for the bisection method): The response for black $\left(7.0 \mathrm{~cd} / \mathrm{m}^{2}\right)$ is defined as $0 \%$, and that for white $(120 \mathrm{~cd} /$ $\mathrm{m}^{2}$ ) is defined as $100 \%$. The axis for each relative cone excitation is also shown. Those for the $L$ and $M$ cones were normalized so that the excitation of the $L$ cone was 0.67 and that of the $M$ cone was 0.33 for the neutral gray of $60 \mathrm{~cd} / \mathrm{m}^{2}$ (this corresponds to the equal energy white point in the cone excitation color diagram). That for the $\mathrm{S}$ cone was arbitrarily normalized so that the excitation of the $S$ cone for the $60 \mathrm{~cd} / \mathrm{m}^{2}$ gray was unity. Each point was from a single session, showing the average of five settings. The results of the 2 observers were very similar.

The input-output function along the achromatic axis was closer to a linear function (solid line) than to a logarithmic function (thin line), as Anstis and Mather (1985) reported. The logarithmic line was defined so that the two points of $(7,0)$ and $(120,100)$ were connected in logarithmic fashion: $R=81 \cdot \log (I)-68$, where $R$ is nor- 
Relative $M$ cone excitation
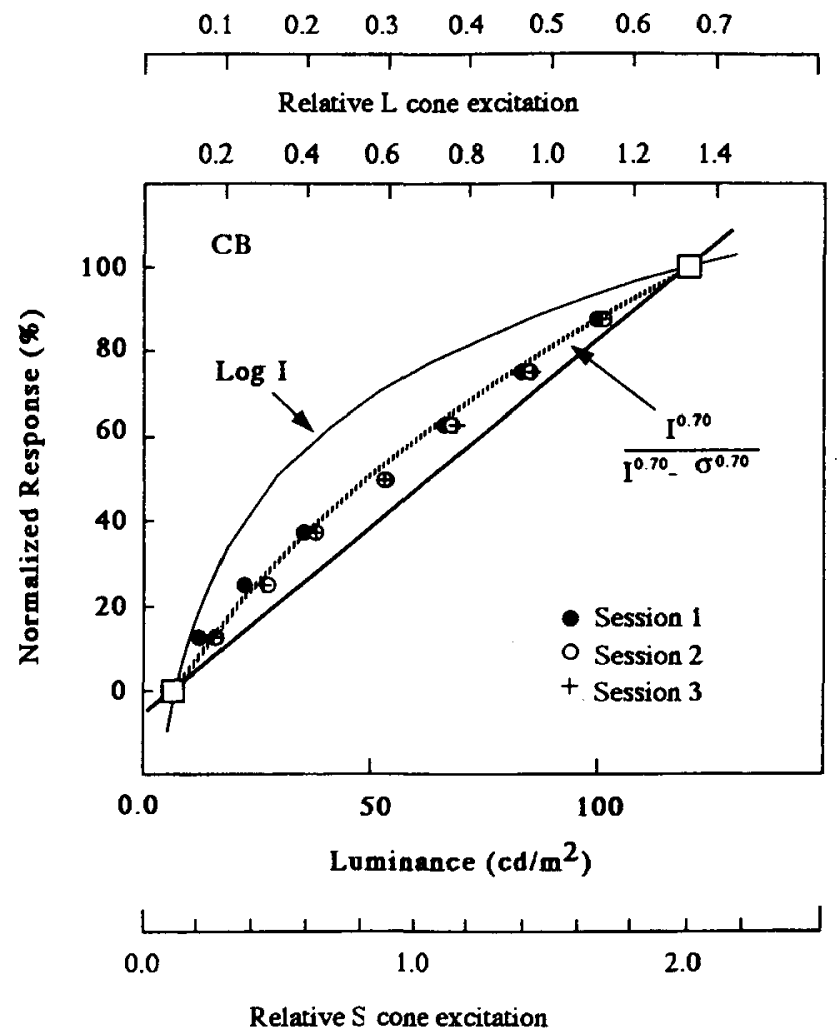

Relative $M$ cone excitation
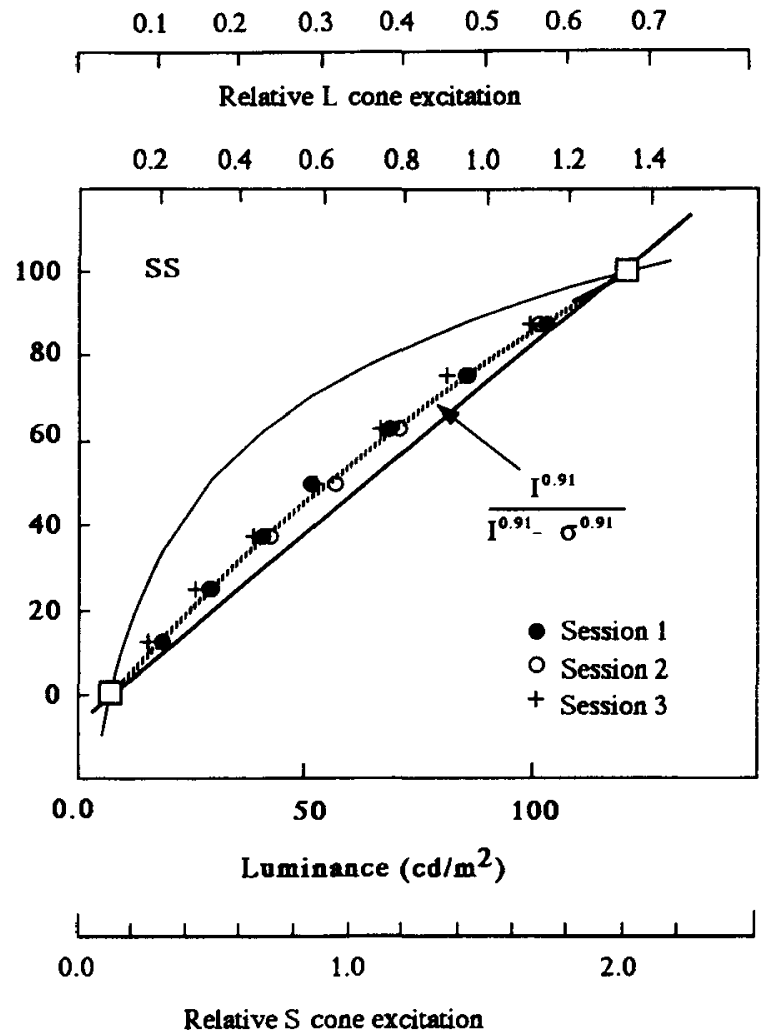

Figure 3. The background luminance of settings (horizontal axis) for seven values of relative response (vertical axis) for the achromatic axis condition. The points for $0 \%$ and $100 \%$ correspond to the black and white of the color pair. The horizontal axis for each cone excitation is also shown (see text). The thick line shows the linear function connecting the black and white values, and the thin line shows the logarithmic function connecting the two points. The broken line is the function proposed by Valeton and van Norren (1983) for cone responses based on physiological responses in monkey.

malized response and $I$ is luminance. However, the data points were consistently to the left of the linear function for both observers over all sessions. This suggests that the input-output function has a small compressive nonlinearity. Indeed, this pattern was also evident in Anstis and Mather. Their luminance bisection data tended to fall below the arithmetic mean of the two bars (see their Figure 4), consistent with a compressive nonlinearity. The nonlinearity is clearly small, however, and we will address; its nature in more detail later.

$S$ cone axis. Figure 4 shows the results for $\mathrm{S}$ cone variations (greenish yellow and purple color pair). The horizontal axis indicates $S$ cone excitation, and the vertical axis shows the normalized response: The response for the greenish yellow is defined as $0 \%$, and that for the purple is defined as $100 \%$. The settings lie to the left of the linear function as in the achromatic axis condition. If we assume no compressive nonlinearity in the color-opponent processes for the yellow/blue color mechanism, the results can be accommodated by a small compressive nonlinearity of the $\mathrm{S}$ cone response.

$\mathbf{L}-\mathbf{M}$ cone axis. Figure 5 shows the result for the equiluminant $\mathrm{L}-\mathrm{M}$ axis (desaturated cyan and magenta color pair). The horizontal axes indicate $\mathrm{L}$ cone and $\mathrm{M}$ cone excitation, and the vertical axis shows the normalized response: The response for desaturated cyan is defined as $0 \%$, and that for desaturated magenta is defined as $100 \%$. The horizontal axes show that the sum of $L$ and $M$ cone responses is always equal to 1.0 , and the excitation of $\mathrm{L}$ cone and $\mathrm{M}$ cone for white is 0.67 and 0.33 , respectively.

The settings lie rightward of the linear function, although the interobserver differences were larger than in previous conditions. The results indicate that more L cone excitation is required in the background to achieve equal effective contrasts of the cyan and magenta bars against the background. It should be noted that the cone modulation for this condition was about six times less than that for achromatic axis (compare the horizontal axis of Figure 3 and that of Figure 5). Therefore, the departure from linearity shown here is in absolute terms much greater than that shown in Figure 3.

\section{NONLINEARITY OF CONE RESPONSE}

The nonlinearities seen for the achromatic and $\mathrm{S}$ cone functions may reflect the compressive cone nonlinearity (Boynton \& Whitten, 1970; Naka \& Rushton, 1966; Valeton \& van Norren, 1983). This is often represented by 

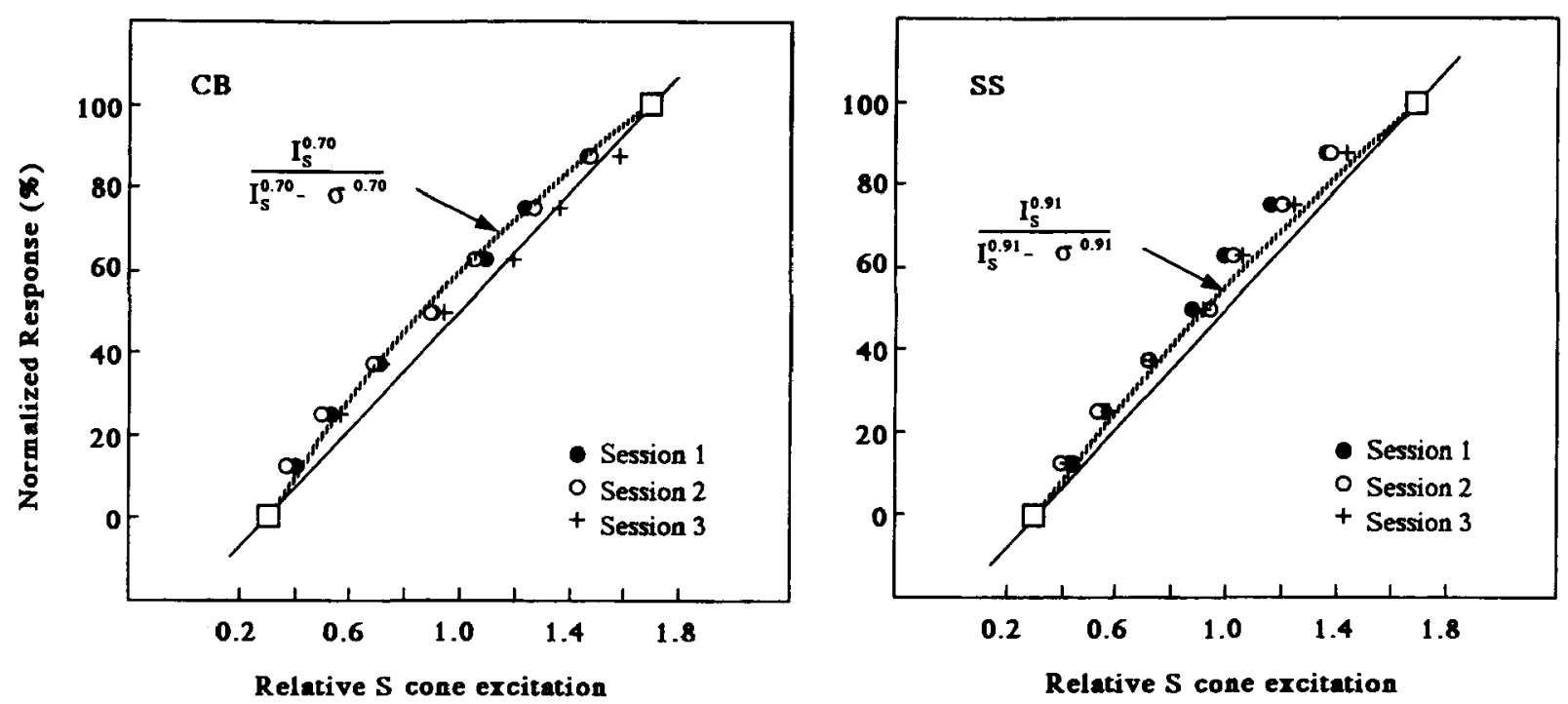

Figure 4. The relative $S$ cone excitation of settings (horizontal axis) for seven values of relative response (vertical axis) for the $S$ cone axis condition. The points for $0 \%$ and $100 \%$ correspond to the greenish yellow and purple of the color pair, respectively. The broken line is the function proposed by Valeton and van Norren (1983).

the Naka-Rushton power function, which can also explain results from human psychophysics (Hood, Finkelstein, \& Buckingham, 1979). The dashed line in Figure 3 shows the function that Valeton and van Norren derived to predict the monkey cone response: $R / R_{\max }=I^{n} /\left(I^{n}+\right.$ $\left.\sigma^{n}\right)$, where $I$ is retinal illuminance in trolands, $\sigma$ is half saturation illuminance, $n$, the exponent, is a free parameter adjusted to fit the data and usually lying between 0.7 and 1 ( 0.74 in their case), $R_{\max }$ is maximum possible cone response, and $R$ is cone response (but not the normalized response between 0 and 100 plotted in Figures 3, 4, and 5).

To apply the cone response model of Valeton and van Norren (1983), we assumed that the adaptation level of their experiment was equivalent to our large surround. The test bars alternated between two fixed values in any given session while the level of the large surround was
Relative M cone excitation

\section{$\begin{array}{lllllll}0.40 & 0.38 & 0.36 & 0.34 & 0.32 & 0.30 & 0.28\end{array}$}

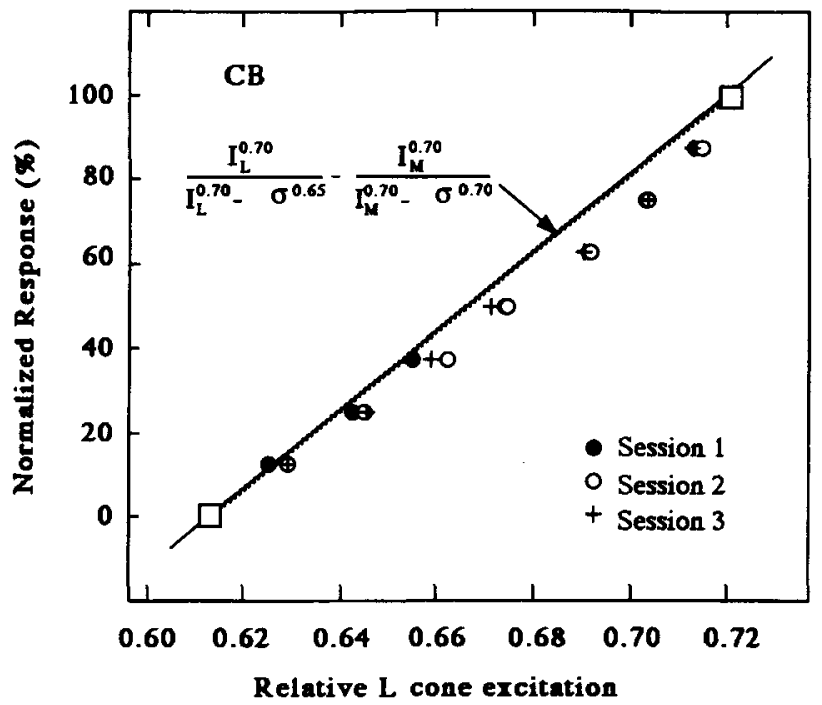

Relative $M$ cone excitation

$$
\begin{array}{lllllll}
0.40 & 0.38 & 0.36 & 0.34 & 0.32 & 0.30 & 0.28
\end{array}
$$

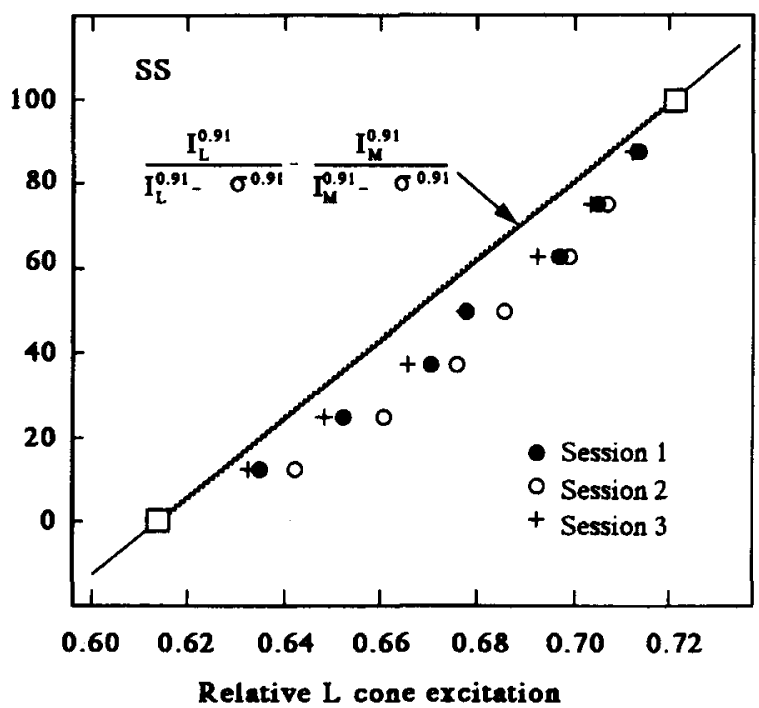

Figure 5. The relative $L$ cone excitation of settings (horizontal axis) for seven values of relative response (vertical axis) for the $L-M$ cone axis condition. The points for $0 \%$ and $100 \%$ correspond to the desaturated magenta and desaturated cyan of the color pair, respectively. $M$ cone excitation is also shown at the top $(M=1-L$ for color changes at equiluminance). The broken line is a prediction of the $L-M$ process based on the function of Valeton and van Norren (1983). 
varied to find the point of equal response to the two tests. Since the two tests held the same value over a session, they could not contribute to any change in adaptation level. On the other hand, Valeton and van Norren used a steady, spatially overlapped background for controlling the adaptation states, so our assumption needs to be examined carefully. Favoring our assumption is the fact that the slight compressive nonlinear characteristics of cone responses are similar at different adaptation levels as long as the stimulus luminance does not differ greatly from the adaptation luminance.

We estimated the retinal illuminance for each of our observers by measuring the pupil size (Wyszecki \& Stiles, 1982 ) as a first approximation: The adaptation field luminance of $60 \mathrm{~cd} / \mathrm{m}^{2}$ corresponds to $380 \mathrm{td}$ for $\mathrm{Ob}-$ server C.B. and 770 td for Observer S.S. Accordingly, $7 \mathrm{~cd} / \mathrm{m}^{2}$ (black) corresponds to $44 \mathrm{td}$ for C.B. and $89 \mathrm{td}$ for S.S., and $120 \mathrm{~cd} / \mathrm{m}^{2}$ (white) corresponds to $760 \mathrm{td}$ for C.B. and 1,530 td for S.S. We estimated the half saturation illuminance as 5,370 for C.B. and 7,080 for S.S. by interpolating between the data for 100-td and 1,000-td backgrounds of Valeton and van Norren's (1983) experiment. We transformed the values of the Naka-Rushton function to match our normalized response values at the lowest and highest values: $R_{\%}=\left[R(I)-R_{\text {black }}\right] /\left(R_{\text {white }}\right.$ $\left.-R_{\text {black }}\right) \times 100$, where $R(I)$ is cone response for luminance $I, R_{\text {black }}$ is the response for black bar luminance, and $R_{\text {white }}$ is the response for white bar luminance. $R_{\%}$ corresponds to the coordinate value in our plots. The best fitting exponent, $n$, was chosen by a least-mean-square procedure minimizing the difference between the predictions and the data for each observer: 0.7 for C.B. and 0.91 for S.S. The value was similar to the value used in previous studies (Boynton \& Whitten, 1970; Naka \& Rushton, 1966; Valeton \& van Norren, 1983). The dashed line in Figure 3 shows the transformed Naka-Rushton function.

The Naka-Rushton functions approximate our achromatic results fairly well, showing less nonlinearity than the logarithmic function. To confirm this, we calculated the average background settings for the 2 observers once their data were transformed to the output values of the linear Naka-Rushton and logarithmic functions. The function that best captures the response properties should linearize the output and therefore give an average of 0.5 for the mid value. The average settings for linear, compressive (Naka-Rushton), and logarithmic functions, respectively, were $0.43,0.49$, and 0.57 for C.B. and $0.47,0.51$, and 0.61 for S.S. The compressive model had the best prediction, and a $t$ test (with Holm's correction for multiple testing) showed that the average was significantly different from 0.5 for linear and logarithmic functions, but not so for the cone compressive function.

To extend the discussion to the $\mathrm{L}-\mathrm{M}$ and $\mathrm{S}$ cone axes, we assumed first that the nonlinearity for the three type of cones is identical and second that the response level (or adaptation level) of the three types of cones is the same at the equal energy white. The first assumption is tentative because the $\mathrm{S}$ cone may be different from the other two (e.g., Mollon, 1982). Nevertheless, we use the same nonlinearity for the $\mathrm{S}$ cone as for $\mathrm{L}$ and $\mathrm{M}$ cones, simply because we do not have much knowledge of the $\mathrm{S}$ cone nonlinearity. The second assumption is supported by the invariance of unique white over a large range of stimulus intensity (Walraven \& Werner, 1991). This invariance requires that the outputs of the three cone classes maintain the same relative proportions as luminance varies, suggesting that all cone responses have a similar nonlinearity (or no nonlinearity). The assumptions provide that $380 \mathrm{td}$ (C.B.) or $770 \mathrm{td}$ (S.S.) corresponds to cone excitation of 1.0 for $S$ cones, 0.67 for $L$ cones, and 0.33 for $\mathrm{M}$ cones. Using these relations, cone excitations for the color pairs used provide the corresponding retinal illuminance values, and, thus, the $R_{\%}$ value for each cone can be derived in the same manner as for the achromatic axis.

The dashed line in Figure 4 shows the calculated response of $S$ cone under these assumptions. The cone response function resembles the experimental result, as it did for the achromatic axis. This suggests that the nonlinearity of the $\mathrm{S}$ cone is indeed similar to that for the $\mathrm{L}$ and $\mathrm{M}$ cones, as we assumed. The nonlinearity for the $\mathrm{S}$ cone axis appears smaller than that for achromatic axis (compare Figures 3 and 4). This is, however, a graphical consequence of the smaller range of cone excitations for the $S$ cone axis. The $S$ cone excitation for the achromatic axis has a range approximately 1.4 times as large as it does for the $S$ cone axis, and both sets of data are fairly well predicted by the same function. However, the data for S.S. show consistently larger nonlinearity than the cone response function. This was not likely due to the difference of $S$ cone from the others. Although the use of different value of exponent, $n$, for $\mathrm{S}$ cone improved the fit, the value of $n$ for the best fit was too small for a cone response function ( 0.47 and 0.41 for C.B. and S.S., respectively). An additional nonlinearity, perhaps from opponent mechanism, may be required to explain the result of S.S.

The same cone nonlinearity was next applied to the analysis of the $\mathrm{L}-\mathrm{M}$ cone axis. We subtracted the excitation of the $M$ cone from that of $L$ cone after the NakaRushton compression. Since the response level of $L$ and $M$ cones are assumed to be the same at the equal energy white, this anchors the $\mathrm{L}-\mathrm{M}$ value for the neutral gray at zero. The model showed only a slight difference from linearity on the scale of the graph (the dashed line in Figure 5). This was first, because the nonlinearities of $\mathrm{L}$ and $M$ cones partially cancel each other and, second, because the range of cone excitation is so narrow that the effect of the Naka-Rushton nonlinearity over this range is small. Clearly, the Naka-Rushton cone nonlinearity falls far short of explaining the empirical nonlinearity seen along the L-M axis. This suggests that a nonlinearity at a second or later (opponent-color) stage is also contributing to the response.

\section{EXPERIMENT 2}

We conducted Experiment 2 to compare the midpoint settings found with the apparent motion method used in Experiment 1 with the midpoint setting based on judging 
contrast directly. If the contrast judgments provide different results, we may be able to differentiate between early nonlinearities that must be common to both tasks and later nonlinearities that could be specific to the contrast or the motion system.

\section{Method}

Experimental conditions were chosen to be similar to those in Experiment 1 except that only the first midpoint setting between the two extremes of each axis was made. No further divisions of the axes followed. Stimuli were presented on a 17-in. RGB monitor (Sony Multiscan $17 \mathrm{GS}$ ), controlled by a video controller (Cambridge Research Systems VSG2/3) with $640 \times 480$ pixels spatial resolution, 12-bit intensity levels per color, and a $120-\mathrm{Hz}$ noninterlaced raster. The red, green, and blue phosphors of the monitor were determined by spectroradiometry to have CIE $x$ and $y$ coordinates of .623 and $.342, .286$ and .605 , and .149 and .065 , respectively. The screen was viewed from a distance of $80 \mathrm{~cm}$ and subtended a visual angle of $21^{\circ} \times 16^{\circ}$. The stimulus configuration was identical to that in Experiment 1 (Figure 1). The outside of the stimulus field $\left(21^{\circ} \times\right.$ $16^{\circ}$ ) was filled with a uniform gray of $45 \mathrm{~cd} / \mathrm{m}^{2}$.

The color pair for the achromatic axis was black of $0.15 \mathrm{~cd} / \mathrm{m}^{2}$ and white of $90 \mathrm{~cd} / \mathrm{m}^{2}$. CIE $x$ and $y$ coordinates of the color pair for the $L-M$ cone axis were .291 and .354 (desaturated cyan) and .374 and .313 (desaturated magenta), and those for the $S$ cone axis were .289 and .237 (purple) and .394 and .455 (greenish yellow). The luminance level for equiluminant color axes was $45 \mathrm{~cd} / \mathrm{m}^{2}$. The $\mathrm{min}-$ imum step of the color change along each axis was limited by the reading of the mouse movement in this experiment, which was 640 steps for each color pair. The equiluminant plane was set by each observer by heterochromatic flicker photometry, as in Experiment 1, but only once before the experimental sessions.

The bars exchanged positions periodically at $1 \mathrm{~Hz}(500 \mathrm{msec}$ for each position) while the observers adjusted the color of the background using a mouse. Two different criteria were used: one based on motion perception, and the other based on apparent contrast of the two bars in the identical stimulation. The first criterion was identical to that in Experiment 1. For the second criterion, the adjustment was made to determine the point at which the two bars appeared to have the same contrast compared to the background. The observers viewed binocularly with natural pupil. Four sessions of five settings in each condition were run by each observer, with randomized order of the conditions. Nine observers with corrected-tonormal acuity participated in the experiment. One of them was one of the authors, who had visual field loss in periphery due to glaucoma developed by the time of the second experiment. We included his data for the analysis since his data were similar to those of the others.

\section{Results}

Table 1 shows the average midpoint settings of the 9 observers with the standard error of the mean for the two criteria for each axis separately. The average within-

Table 1

Settings of Equal Motion and of Equal Apparent Contrast in Experiment 2 With the Within-Observer Difference Between Them (Averages and Standard Errors Over 9 Observers)

\begin{tabular}{|c|c|c|c|c|c|c|}
\hline & \multicolumn{2}{|c|}{ Motion } & \multicolumn{2}{|c|}{ Contrast } & \multicolumn{2}{|c|}{$\begin{array}{c}\text { Difference } \\
\text { (Motion - Contrast) }\end{array}$} \\
\hline & Average & $S E$ & Average & $S E$ & Average & $S E$ \\
\hline Achromatic & .415 & .019 & 440 & .019 & -.026 & .009 \\
\hline L-M cone & .577 & .014 & .516 & .011 & .061 & .018 \\
\hline S cone & .467 & .010 & .450 & .008 & .019 & .007 \\
\hline
\end{tabular}

observer differences for the two criteria are also shown. The values are in terms of the percentage of the separation between the colors of the two bars, with $0 \%$ taken as the darker bar, the greenish yellow bar, and the cyan bar for the achromatic, S cone, and L-M cone axes, respectively. Midpoint settings falling below 0.5 indicate that less physical contrast of the darker bar, the greenish yellow bar, or the cyan bar against the background was required to match the effective contrast of the other bar. The results for the motion criterion were consistent with the results of Experiment 1. First, a compressive nonlinearity was found for achromatic and $\mathrm{S}$ cone axes. The midpoint gray, for example, required less luminance than the arithmetic mean of the light and dark bars. Second, for the $\mathrm{L}-\mathrm{M}$ axis, more $\mathrm{L}$ cone excitation was required (less contrast between the magenta bar and background) in the background to achieve equal effective contrasts of the cyan and magenta bars. This was the same result as found in Experiment 1.

The results for the perceived contrast criterion show departures from linear midpoint settings for the achromatic and $\mathrm{S}$ cone axes, which were similar in magnitude and direction to those for the motion criterion. However, the results for the $\mathrm{L}-\mathrm{M}$ cone axis were much closer to 0.5 , the linear midpoint. A $t$ test with Holm's correction for multiple testing showed that all the settings except this one differed significantly from 0.5 . The difference between the motion and contrast criteria was largest for the L-M settings. A $t$ test with Holm's correction for paired comparisons of settings between motion and contrast also showed significant difference only for the $\mathrm{L}-\mathrm{M}$ axis. This suggests that the nonlinearity for the $\mathrm{L}-\mathrm{M}$ axis seen in Experiment 1 and here with the motion criterion is specific to apparent motion or transient mechanism(s), whereas that for the other axes is likely a general characteristic of the early visual system.

\section{DISCUSSION}

The Anstis and Mather (1985) apparent-motion technique revealed nonlinear coding for all three axes: achromatic, $\mathrm{S}$ cone, and $\mathrm{L}-\mathrm{M}$. The slight nonlinearities of the achromatic and $S$ cone axes are well modeled by a compressive nonlinearity of cone response (Boynton \& Whitten, 1970; Naka \& Rushton, 1966; Valeton \& van Norren, 1983). A much stronger nonlinearity, possibly arising in the color-opponent processes, is necessary to explain the results for $\mathrm{L}-\mathrm{M}$ cone axis. Experiment 2 demonstrated that this nonlinearity was specific to the $\mathrm{L}-\mathrm{M}$ contribution to apparent motion. A logarithmic nonlinearity was not appropriate for any axis, being too severe for the achromatic and $\mathrm{S}$ cone axes and insufficient for the $\mathrm{L}-\mathrm{M}$ cone axis.

In this section, we discuss motion responses to color, responses in the different axes, the differences in results between the $\mathrm{S}$ cone axis and the $\mathrm{L}-\mathrm{M}$ cone axis, the effect of nonlinearity of contrast coding, and, finally, the possible effects of light adaptation and color adaptation. 
The results for the $\mathrm{S}$ cone axis, like those for the achromatic stimuli, could be predicted by the Naka-Rushton nonlinearity of cone responses. Our $\mathrm{S}$ cone axis results reflect the combined nonlinearities from higher level mechanisms as well as cones. Our results therefore suggest that the nonlinearities that might be occurring in the blue/ yellow color-opponent mechanism are largely outweighed by the nonlinearity of S cone responses. Along the L-M cone axis, however, the effect of the Naka-Rushton cone nonlinearity is far less than the observed nonlinearity, and an additional nonlinearity at the level of the red/green contribution to motion mechanisms must be considered.

These results do not rule out the contribution of cone nonlinearities to the $\mathrm{L}-\mathrm{M}$ cone stimuli nor that of the nonlinearity of opponent-color processes to $S$ cone stimuli. First, the experimental results along the L-M cone axis undoubtedly include the same cone nonlinearity measured for these cones in the achromatic condition. It is simply overwhelmed by the larger subsequent nonlinearity at the next level in the motion pathway. Second, a close look at the results along the $\mathrm{S}$ cone axis (Figure 4) reveals that the experimental data do possibly show a slightly larger nonlinearity than that predicted by cone nonlinearity. This may indicate a small nonlinear contribution at the opponent level for the $\mathrm{S}$ cone axis, although the nonlinearity is much smaller than that for $\mathrm{L}-\mathrm{M}$ cone axis (remember the large difference between the two conditions in cone contrast). Since our task involves motion perception, the $\mathrm{S}$ cone or $\mathrm{S}$ cone pathway (Wisowaty \& Boynton, 1980) may not have the temporal resolution to respond to the stimulus. However, our stimulus was constructed to differentially modulate only $S$ cones, and compelling motion was seen, with no obvious qualitative differences compared with the other stimuli. Moreover, psychophysical evidence suggests that the temporal resolution for $\mathrm{S}$ cone is actually not unlike that for $\mathrm{L}$ and $\mathrm{M}$ cones (Stockman, MacLeod, \& DePriest, 1991). Temporal impulse functions measured for hue substitution also revealed that there is little difference for different directions of hue shift (Uchikawa \& Ikeda, 1986). These results suggest that there is not much difference either among the temporal properties of three types of cones or among the temporal properties of the red/green and blue/yellow mechanisms. Our finding of a much smaller nonlinearity for the $\mathrm{S}$ cone secondary processes relative to the $\mathrm{L}-\mathrm{M}$ cone secondary processes is therefore puzzling.

It is interesting to compare our results with those of tests of linearity in color space with other techniques. In particular, the degree of nonlinearity is often assumed to be larger for the yellow/blue system than for the red/ green system, on the basis of results in hue cancellation experiments (e.g., Ayama \& Ikeda, 1989). The chromatic valence functions derived from these results can be expressed by a linear sum of cone outputs for the red/green but not for the yellow/blue mechanism. In contrast, in our study, we found a more substantial nonlinearity for the $\mathrm{L}-\mathrm{M}$ axis, which is closely related to the red/green color opponent system, than for the S cone axis. However, the nonlinearity we report for the $\mathrm{L}-\mathrm{M}$ axis is prominent only when motion criterion was used. There was little deviation from linearity when the observers matched the apparent contrast of the two bars. We suggest that our L-M nonlinearity is specific to motion or transient mechanisms.

On the other hand, if we consider the Munsell system (a uniform color space), it is nonlinear in CIE $x, y$ coordinates in all directions (Wyszecki \& Stiles, 1982), and this holds in cone excitation coordinates as well because they are a linear transformation of CIE $x, y$ coordinates. To see the amount of deviation from linearity, we calculated the coordinates of 64 Munsell chips of Munsell Value 6 in the cone excitation diagram with a hypothetical equal energy white illuminant. These chips were chosen to cover the lightness plane of Value 6 (even-number chromas for 20 hues). Equal chroma contours were drawn in the cone excitation diagram, from which chroma versus $\mathrm{L}$ or $\mathrm{S}$ cone excitation function was obtained. The functions show that the deviation is slight and the amount of the nonlinearity is similar for the $\mathrm{L}-\mathrm{M}$ and $\mathrm{S}$ cone axes in the range of the colors used in our experiments. This suggests that the larger nonlinearity that we found for the $\mathrm{L}-\mathrm{M}$ cone axis is not directly related to the perception of color per se. This again agrees with the results in Experiment 2 in which the large nonlinearity for $\mathrm{L}-\mathrm{M}$ cone axis for motion task was not found in the contrast task.

In addition to the cone nonlinearity, there is also a wellestablished nonlinearity of contrast coding around the adapting value. Specifically, the sensitivity for discrimination of contrast differences is higher when the test stimuli are similar to the background, an effect that is called crispening (Kaneko, 1964; Legge \& Kersten, 1983; Takasaki, 1966; Whittle, 1986, 1992). This effect corresponds to a local increase of the slope in the input-output function, with the steepest slope at the background level. We did not find any indication of this sigmoid shape in our data. This is, perhaps, because the technique used in our experiment cannot evaluate the linearity in the contrast domain. Comparison was made between the two bars of opposite contrasts against the background in the technique, and, therefore, no effect was expected due to the nonlinearity found using stimuli of the same polarity with different contrasts.

Finally, we do not feel that adaptation is the source of the larger L-M effect. We used a large adaptation field of constant luminance and chromaticity that surrounded the test field to minimize the change of adaptation states of luminance and chrominance of the test field. However, there are well-known, rapid, local adaptation effects in the visual system (MacLeod \& He, 1993; MacLeod, Williams, \& Makous, 1992) in addition to classical slow adaptation effects. A $500-\mathrm{msec}$ flash of light, for example, is long enough to produce an adaptation effect even with a large uniform surround. The time course of the response in the experiment of Valeton and van Norren (1983), for example, shows that response stabilizes less than $100 \mathrm{msec}$ after the onset of 150 -msec presentation of the stimulus (their Figure 2a). These rapid adaptation effects are therefore present in the measurements of cone responses, 
including those we used to model the nonlinearity in our achromatic and $\mathrm{S}$ cone results. Our data, therefore, include the appropriate local adaptation effect.

\section{Conclusion}

In the apparent-motion task, all of the cardinal color axes showed some deviation from linearity. For the $S$ cone and achromatic stimuli, the nonlinearity was small and was adequately predicted by the Naka-Rushton compressive nonlinearity of cone responses. The small deviations from linearity demonstrate both that the early nonlinearities are clearly not logarithmic and that the motion response for these two axes can be described as a reasonably linear response to contrast modulation along their axes.

However, the nonlinearity for the L-M axis color pair was substantially larger than the combined effects of the two small cone nonlinearities. We therefore suggested a contribution of a strong nonlinearity at a second, coloropponent site that becomes noticeable only at higher temporal rates or perhaps only for apparent-motion analyses.

\section{REFERENCES}

Anstis, S. M., \& MATHER, G. (1985). Effect of luminance and contrast on direction of ambiguous apparent motion. Perception, 14, 167-179.

Ayama, M., \& IKEDA, M. (1989). Dependence of the chromatic valence function on chromatic standards. Vision Research. 29, 1233-1244.

Boynton, R. M. A. (1986). A system of photometry and colorimetry based on cone excitations. Color Research \& Application, 11, 244-252.

Boynton R. M., \& Whitten, D. N. (1970). Visual adaptation in monkey cones: Recording of late potentials. Science, 170, 1423-1426.

Delboeuf, J. (1883). Elements de psychophysique, generale et speciale [Elements of psychophysics, general and particular]. Paris: Ballière et Cie.

Derrington A. M., Krauskopf, J., \& Lennie, P. (1984). Chromatic mechanisms in lateral geniculate nucleus of macaque. Journal of Physiology, 357, 241-265.

Fechner, G. T. (1966). Elements of psychophysics (H. Adler, Trans.). New York: Holt, Rinehart \& Winston. (Original work published 1860).

Flanagan, P., Cavanagh, P., \& Favreau, O. E. (1990). Independent orientation-selective mechanisms for the cardinal directions of colour space. Vision Research, 30, 769-778.

Hood, D. C., Finkelstein, M. A., \& Buckingham, E. (1979). Psychophysical tests of models of the response function. Vision Research, 29, 401-406.
KaneKo, T. A. (1964). Reconsideration of the Cobb-Judd lightness function. Acta Chromatica, 1, 103-110.

LeGGe, G. E., \& KERSTEN, D. (1983). Light and dark bars: Contrast discrimination. Vision Research, 23, 473-483.

LehmanN, A. (1886). Über die Anwendung der Methode der mittleren Abstufungen auf den Lichtsinn. Philosophische Studien, 3, 497-533.

MACLEOD, D. I. A., \& BoynTON, R. M. (1979). A chromaticity diagram showing cone excitation by stimuli of equal luminance. Journal of the Optical Society of America, 69, 1183-1186.

MACLEOD, D. I. A., \& HE, S. (1993). Visible flicker from invisible patterns. Nature, 21, 256-258

Macleod D. I. A., Williams, D. R., \& Makous, W. (1992). A visual nonlinearity fed by single cones. Vision Research. 32, 347-363.

Mollon, J. D. (1982). Color vision. Annual Review of Psychology, 33, 41-85.

NAKA, K.-I., \& RushTON, W. A. H. (1966). S-potential from colour units in the retina of fish (Cyprinidae). Journal of Physiology, 185, 536-555.

Plateau, J. (1872). Sur la mesure des sensations physiques, et sur la loi qui lie l'intensité de ces sensations à l'intensité de la cause excitante [On the measurement of physical sensations and the law that links the intensity of these sensations to the intensity of the stimulation]. Bulletins de l'académie royale de Belgique, 33, 376-389

Stockman, A., Macleod, D. I. A., \& DePriest, D. D. (1991). The temporal properties of the human short-wave photoreceptors and their associated pathways. Vision Research, 31, 189-208.

TAKASAKI, H. (1966). Lightness change of grays induced by change in reflectance of gray background. Journal of the Optical Society of America, 56, 504-509.

UCHIKAWA, K., \& IKEDA, M. (1986). Temporal integration of chromatic double pulses for detection of equal-luminance wavelength changes. Journal of the Optical Society of America A, 3, 2109-2115.

VAleton, J. M., \& VAN NORREN, D. (1983). Light adaptation of primate cones: An analysis based on extracellular data. Vision Research, 23, 1539-1547.

WALRAVEN, J., \& WERNER, J. S. (1991). The invariance of unique white: A possible implication for normalizing cone action spectra. Vision Research, 31, 2185-2193.

WisowatY, J. J., \& Boynton, R. M. (1980). Temporal modulation sensitivity of the blue mechanism: Measurements made without chromatic adaptation. Vision Research, 20, 895-909.

WhitTle, P. (1986). Increments and decrements: Luminance discrimination. Vision Research, 26, 1677-1691.

WhitTLE, P. (1992). Brightness, discriminability and the "crispening effect." Vision Research, 32, 1493-1507.

Wyszecki, G., \& Stiles, W. S. (1982). Color science (2nd ed.) New York: Wiley.

(Manuscript received March 17, 1998; revision accepted for publication November 4,1999 .) 